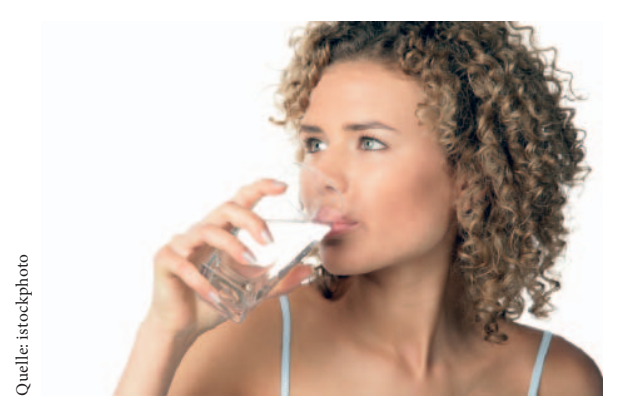

Viel zu trinken, ist ein Ratschlag, den Ärzte gerne erteilen. Genügend Wasser im Laufe des Tages soll dazu führen, dass die Nieren funktionieren, der Flüssigkeitshaushalt nicht aus dem Gleichgewicht gerät und der Körper leistungsfähig bleibt.

Die britische Ärztin Margaret McCartney hält allerdings nicht viel von solchen Tipps, und in einem Beitrag für das British Medical Journal (BMJ 2011;343:d4280) legte sie im Frühjahr des Jahres eine grundsätzliche Kritik dar. Es sei vollkommen unklar, auf welcher wissenschaftlichen Basis die Trinkempfehlungen beruhten. Studien, die eine positive Auswirkung der Zufuhr von 1,5-2 Liter pro Tag auf die Gesundheit der Bevölkerung belegten, gebe es nicht. Hinter den vielen Promotoren des ausreichenden Trinkens stünden Interessengruppen, wie z.B. die Hersteller von Mineralwasser. Wissenschaftliche Konferenzen über Hydratisierung und Gesundheit würden von ihnen gesponsert.

McCartneys Kritik ist nur der jüngste und an sehr prominenter Stelle erschienene Zweifel an den Trinkempfehlungen. Sie wurden von der Presse stark beachtet und sorgten vor allem in Grossbritannien für eine öffentliche Diskussion der Fachleute.

In den letzten Jahren sind bereits mehrfach Studien erschienen, die sie in einem anderen Licht erscheinen lassen. So haben sich auch die amerikanischen Nephrologen Dan Negoianu und Stanley Goldfarb von der University of Pennsylvania in Philadelphia im Fachblatt Journal of the American Society of Nephrology (J Am Soc Nephrol 2008;19:1041-1043) mit dem

DOI: 10.1159/000331644

\title{
Lieber nicht viel trinken
}

Der Ratschlag, viel zu trinken, um den Körper gesund und leistungsfähig zu halten, ist in die Kritik geraten. Nicht fundiert und womöglich gefährlich sei er.

Thema auseinandergesetzt. Sie haben sich auf die Suche nach den Quellen der heute international weitverbreiteten 1,5- bis 2-Liter-Empfehlung gemacht und sind dabei nicht fündig geworden. Die wissenschaftliche Grundlage sei nicht vorhanden, argumentieren die Ärzte. Zwar stimme es, dass das Wasser die Nierenfunktion anrege; dass dies jedoch einen positiven Effekt auf die Gesundheit habe, sei nicht bestätigt. Lediglich beim Versuch, Nierensteine herauszuschwemmen, gebe es einen wirklichen Nutzen des vielen Trinkens. Dass vermehrtes Trinken gegen Migränekopfschmerzen hilft, ist nach den Angaben der beiden Nephrologen bisher nur in einer einzigen Arbeit untersucht worden. Das Ergebnis: Ein Zusammenhang zwischen Trinkmenge und Kopfschmerzen lasse sich nicht finden. Dass viel Wasser vor Falten bewahrt, sei bisher von keiner Studie gestützt worden. Auf ebenso tönernen Füssen stehe die Behauptung, dass das Wassertrinken beim Abnehmen helfe. Einen Beleg, dass das Völlegefühl den Appetit dämpfe, gebe es nicht.

Mittlerweile sind die Empfehlungen längst zum Allgemeingut im Wissen der Bevölkerung über einen gesunden Lebenswandel geworden. Nachdem sich Patienten früher immer an die Regel erinnern mussten, auch tatsächlich 1,5-2 Liter Wasser pro Tag zu trinken, kann sich der moderne Mensch der Unterstützung der Technik bedienen: Auf dem Smartphone lassen sich Apps laden, die einen in regelmässigen Abständen daran erinnern, mal wieder ein Glas Wasser zu trinken.
In der Diskussion ist bisher auch übersehen worden, dass zu viel Flüssigkeit ohne Weiteres auch schädlich sein kann. Aus dem Leistungs- und Ausdauersport weiss man, dass eine erhöhte Zufuhr an Wasser unter Umständen $\mathrm{zu}$ einer viel $\mathrm{zu}$ geringen Konzentration von Natrium im Blut führen kann. Die Hyponatriämie kann zur Ansammlung von Wasser in den Organen führen. Im schlimmsten Fall kann sie sogar eine tödliche Hirnschwellung hervorrufen. Dass diese Gefahr tatsächlich gegeben ist, zeigt eine Untersuchung von Tim Noakes vom Institut für Sportwissenschaften der Universität Cape Town, Südafrika. Er hat im British Journal of Sports Medicine (Br J Sports Med 2010;44: 998-1001) eine Studie veröffentlicht, in der er belegt, dass die Häufigkeit von sportbedingten Hyponatriämien in den letzten 10 Jahren stark zugenommen hat. Er stellt eine direkte Verbindung zu der Empfehlung von sportmedizinischen Fachgesellschaften her, die um die Jahrtausendwende empfohlen hatten, viel zu trinken, wenn man bei Sport stark schwitzt. Wie seine Untersuchungen belegen, hat mehr als jeder 10. Teilnehmer eines Marathonlaufs erste Anzeichen einer Hyponatriämie. Auch andere Wissenschaftler kommen zu ähnlichen Schlussfolgerungen. Selbst bei schweisstreibenden Sportarten, wie etwa einem Marathonlauf, solle man nur so viel trinken, wie man Durst habe - aber nicht mehr. Das empfiehlt jetzt die «Marathon Medical Directors Association». Auch im Alltag sei der Durst der beste Ratgeber, wann man etwas trinken solle. $(r f i)$ 